\title{
ERRATUM
}

\section{Erratum to: The contribution of cell phenotype to the behavior of gastric cancer}

\author{
Enrico Solcia $\cdot$ Catherine Klersy $\cdot$ Alessandro Vanoli • \\ Federica Grillo $\cdot$ Rachele Manca $\cdot$ Francesca Tava $\cdot$ \\ Ombretta Luinetti $\cdot$ Roberto Fiocca
}

Published online: 15 March 2013

(C) The International Gastric Cancer Association and The Japanese Gastric Cancer Association 2013

\section{Erratum to: Gastric Cancer \\ DOI 10.1007/s10120-012-0208-8}

The colored lines in the Kaplan-Meier curves in Fig. 2 are not clearly differentiated when printed in black and white. A modified figure, more readily understandable, is shown here.

The online version of the original article can be found under doi:10.1007/s10120-012-0208-8.

E. Solcia $\cdot$ A. Vanoli $\cdot$ R. Manca $\cdot$ F. Tava $\cdot$ O. Luinetti Department of Pathology, University of Pavia, Fondazione Istituto di Ricovero e Cura a Carattere Scientifico (IRCCS)

Policlinico San Matteo, 27100 Pavia, Italy

C. Klersy

Biometry and Statistics Unit, Fondazione IRCCS Policlinico

San Matteo, 27100 Pavia, Italy

F. Grillo $\cdot$ R. Fiocca $(\bowtie)$

Department of Pathology, University of Genova,

IRCCS Azienda Ospedaliera Universitaria San Martino IST,

Via De Toni 14, 16132 Genova, Italy

e-mail: fiocca@unige.it

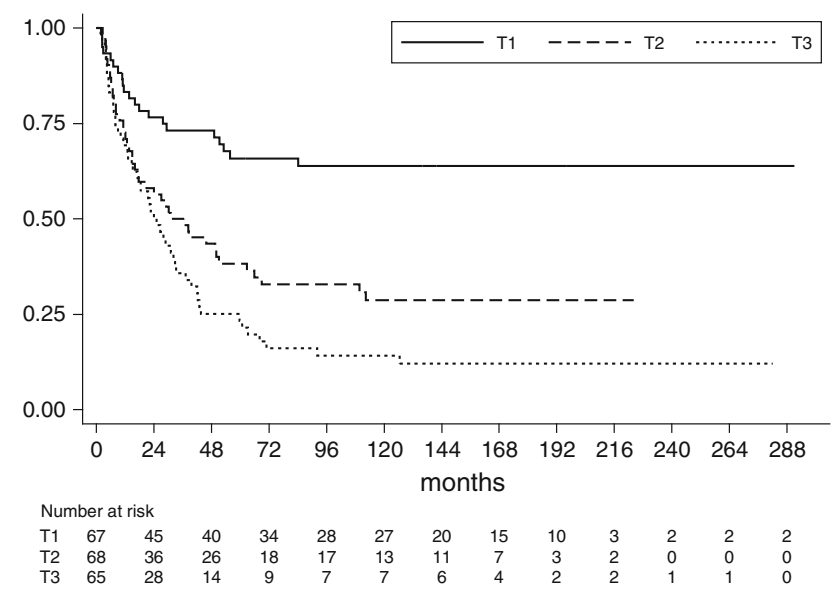

Fig. 2 Kaplan-Meier survival estimate, by tertiles of biomarker burden 